\title{
EVAPOTRANSPIRACIÓN DEL CULTIVO DE TOMATE DE CÁSCARA (Physalis ixocarpa Brot.) ESTIMADA MEDIANTE EL POTENCIAL MÁTRICO DEL SUELO
}

\author{
HUSK TOMATO (Physalis ixocarpa Brot.) CROP EVAPOTRANSPIRATION ESTIMATED \\ BY SOIL MATRIC POTENTIAL
}

\author{
Rutilo López López ${ }^{\text {1* }}$, Ramón Arteaga Ramírez², Mario A. Vázquez Peña ${ }^{3}$, \\ Irineo L. López $\mathrm{Cruz}^{2}$ e Ignacio Sánchez Cohen ${ }^{3}$
}

${ }^{1}$ Instituto Nacional de Investigaciones Forestales Agrícolas y Pecuarias (INIFAP), Campo Experimental Huimanguillo. Km. 1 Carretera Huimanguillo-
Cárdenas. Huimanguillo, Tabasco, México. 86400, Tel. 01 (917) 375-0516, Fax 01 (917) 375-0397. ${ }^{2}$ Postgrado en Ingeniería Agrícola y Uso Integral del
Agua, Universidad Autónoma Chapingo. Km. 38.5 Carretera México-Texcoco. 56230, Chapingo, Texcoco, México. ${ }^{3}$ Centro Nacional de Investigación
Disciplinaria en Relaciones Agua Suelo Planta Atmósfera, INIFAP. Km. 6.5 Canal Sacramento, Zona Industrial Gómez Palacio. 35150, Durango, México.

* Autor para correspondencia (lopez.rutilo@inifap.gob.mx)

\section{RESUMEN}

La poca disponibilidad de agua observada en algunas presas del país en los últimos años y la sobreexplotación de los acuíferos subterráneos hacen urgente el establecimiento de estrategias para hacer un uso racional y eficiente de este recurso. Una estrategia es la optimización del riego mediante el cálculo de las necesidades hídricas para la programación de riegos de los cultivos. En el presente estudio los objetivos fueron determinar los coeficientes de cultivos (Kc) para el tomate de cáscara (Physalis ixocarpa Brot.) con y sin cobertura plástica, $\mathbf{y}$ determinar el efecto de láminas de riego y acolchado plástico en el potencial mátrico del suelo a diferentes profundidades. Se generó una ecuación de regresión que relaciona los datos del potencial mátrico del suelo con la pérdida de humedad medida con el lisímetro, y con esta ecuación se estimó la evapotranspiración del cultivo (ETc). Los tratamientos del experimento consistieron de cinco niveles de láminas de riego $(40,60,80$, 100 y $120 \%$ de la evapotranspiración de referencia, ET 0 ), y dos niveles de acolchado, con y sin plástico. La ETo se utilizó como base para calcular la lámina de riego por medio del goteo. Los resultados mostraron que con $60 \%$ de la $\mathrm{ET}_{0}$, expresada en lámina de riego, mantiene niveles de potencial mátrico cercanos a capacidad de campo $(<-30 \mathrm{kPa})$ en las distintas capas del suelo con $y$ sin acolchado plástico. Durante la etapa reproductiva los potenciales bajaron hasta alcanzar valores de $\mathbf{- 8 0} \mathbf{~ k P a}$, incluso con $100 \%$ de la ET, lo cual se utilizó para estimar y ajustar los Kc. Sin acolchado los Kc fueron: $0.3,1.1$ y 0.86 para las etapas vegetativa, reproductiva y maduración respectivamente. También se determinó que los Kc pueden estimarse a partir de mediciones del índice de área foliar (IAF) de un cultivo creciendo con máxima disponibilidad de agua.

Palabras clave: Physalis ixocarpa, evapotranspiración, programación de riegos, acolchado plástico, índice de área foliar.

\section{SUMMARY}

Due to low water availability in some Mexican dams, as reported during the last years, and to over-exploitation of ground-water aquifers, it is necessary to design strategies for rational and efficient water use. Optimization of irrigation by calculating crop water re- quirements to schedule irrigation is one strategy. The objectives of this work were to determine crop coefficients (Kc) for husk tomato (Physalis ixocarpa Brot.) under two conditions, with and without plastic mulching, and to observe the effect of irrigation watering rates combined with mulching on the water matric potential at different soil depths. A linear regression model between soil matric potentials and moisture losses, as measured with a lysimeter, was generated, and this model was used to estimate crop evapotranspiration (ETc) for the growing season. Five levels of water irrigation $(40,60,80,100$ and $120 \%$ of reference evapotranspiration, ET 0 ) combined with two conditions of plastic mulching (with and without) were tested. ETo was used to estimate irrigation demand and applied by a drip tape irrigation system. Results showed that $60 \%$ of the ETo, expressed as supplied irrigation water, keeps the soil matric water potential level near field capacity $(<-30 \mathrm{kPa})$ at different soil depths, both for mulching and no mulching. During the crop reproductive phase, the soil matric potentials decreased to $-80 \mathrm{kPa}$, even in plots watered with $100 \%$ of the ET; this result was used to estimate and adjust the formerly estimated Kc. Without plastic mulching Kc's were $0.3,1.1$ and 0.86 for the vegetative, reproductive and maturity phases, respectively. Kc could also be estimated from leaf area index (LAI) values measured on crops growing under maximum water availability.

Index words: Physalis ixocarpa, evapotranspiration, irrigation scheduling, plastic mulching, leaf area index.

\section{INTRODUCCIÓN}

La poca disponibilidad de agua observada en algunas presas de la República Mexicana en los últimos años y la sobreexplotación de los acuíferos subterráneos hacen urgente el establecimiento de estrategias para hacer un uso racional y eficiente de este recurso. Entre estas estrategias destaca la tecnificación de los sistemas de riego para mejorar su eficiencia y rentabilidad (Catalán y Villa, 2009). La programación del riego es un proceso de decisión orientado a determinar las cantidades de agua por 
aplicar y las fechas de aplicación de cada riego, para minimizar deficiencias o excesos de humedad en el suelo que pudieran causar efectos adversos en el crecimiento, rendimiento y calidad de los cultivos. La programación de los riegos normalmente se ejecuta sin soporte técnico alguno, sino únicamente con base en la experiencia de los productores.

Las necesidades hídricas del cultivo se relacionan con la evapotranspiración (ET), proceso del sistema suelo-planta que combina la pérdida de agua del suelo por evaporación y de la planta por transpiración (Burman y Pochov, 1994). Según la FAO (Allen et al., 1998), la evapotranspiración de referencia $\left(\mathrm{ET}_{0}\right)$ se define como la máxima cantidad de agua que pierde un cultivo hipotético de referencia de una altura de $0.12 \mathrm{~m}$, una resistencia de la superficie de $70 \mathrm{~s} \mathrm{~m}^{-1} \mathrm{y}$ un albedo de $0.23 \mathrm{~m}$, similar a la ET que ocurre en una superficie extensa de pasto verde de altura uniforme, que crece activamente y que está bien regada.

Para estimar la evapotranspiración de un cultivo específico se necesita considerar al propio cultivo y a las condiciones ambientales. Las condiciones meteorológicas determinan la demanda evaporativa, mientras que el dosel del cultivo y la humedad del suelo determinan la magnitud en que la demanda será satisfecha. La ET de un cultivo puede ser estimada si están disponibles las mediciones o estimaciones de $\mathrm{ET}_{0}$. Estas mediciones o estimaciones representan la demanda meteorológica, y los coeficientes del cultivo representan la habilidad de las plantas y del suelo para satisfacer esa demanda (Jensen y Wright, 1978).

Entre los métodos usados para medir la evapotranspiración en una superficie cultivada destaca el del lisímetro que mide la evaporación del suelo desnudo o la evapotranspiración del área sembrada con un cultivo. Los lisímetros son grandes depósitos llenos de suelo, generalmente instalados en el campo para representar las condiciones ambientales naturales, y en el que las condiciones del sistema agua-suelo-planta pueden regularse a conveniencia y medirse con más precisión que en el perfil natural de suelo (Hillel, 1980). Este método aporta una medición directa de la evapotranspiración del cultivo, y se usa frecuentemente para el estudio de efectos climáticos y para evaluar los métodos de estimación.

Cuando no se dispone de lisímetro, comúnmente se usa el método del balance hídrico en campo, el cual permite calcular la evapotranspiración real del cultivo (ETc) y comprueba que ésta estima la pérdida de humedad en el sistema suelo-planta-atmósfera, importante en la programación del riego (Lubana et al., 2001).
Se han utilizado diversos métodos experimentales para obtener la $\mathrm{ET}_{0}$ a partir de la información meteorológica y la $\mathrm{ET}_{\mathrm{c}}$ a partir de coeficientes de cultivo $(\mathrm{Kc})$, métodos que han generado diferentes tipos de curvas (Doorenbos y Pruitt, 1977; Jensen, 1981; Burman y Pochov, 1994; Allen et al., 1998; Dodds et al., 2005). En este trabajo, se postula la hipótesis que con los valores del potencial mátrico del suelo en condiciones de máxima disponibilidad de agua se puede estimar la evapotranspiración, y con ésta los coeficientes del cultivo de tomate de cáscara (Physalis ixocarpa Brot.).

Los objetivos del estudio fueron: 1) Determinar los coeficientes de cultivos $(\mathrm{Kc})$ mediante el potencial mátrico del suelo y pérdida de humedad del suelo medida en el lisímetro; 2) Proponer una manera alternativa para el cálculo del Kc a partir del índice de área foliar; y 3) Conocer el efecto de la lámina de riego sobre el potencial mátrico a diferentes profundidades del suelo con y sin acolchado plástico, en tres etapas fenológicas del cultivo de tomate de cáscara (vegetativa-floración; floraciónfructificación; y maduración-senescencia).

\section{MATERIALES Y MÉTODOS}

\section{Sitio experimental y material genético utilizado}

El estudio se efectuó en Chapingo, Estado de México, situado geográficamente a $19^{\circ} 29^{\prime} \mathrm{LN}$ y $98^{\circ} 53^{\prime} \mathrm{LO}$, y a una altitud de $2250 \mathrm{~m}$. El clima de la localidad es del tipo $\mathrm{Cb}(\mathrm{Wo})(\mathrm{W})(\mathrm{i}) \mathrm{g}$, que corresponde a un templado subhúmedo con lluvias en verano, una época seca en invierno, y con oscilación térmica pequeña entre 5 y $7{ }^{\circ} \mathrm{C}$ (Arteaga et al., 2006). La temperatura media anual es de $15.5^{\circ} \mathrm{C}$, con mayo como el mes más caliente y enero el más frío. La precipitación media anual es de $664 \mathrm{~mm}$.

Se cultivó tomate de cáscara con sistema de riego por goteo durante el periodo comprendido entre marzo a junio de 2007. El material vegetal que se utilizó fue la variedad 'CHF1-Chapingo' generada en la Universidad Autónoma Chapingo.

\section{Producción de plántulas y trasplante}

La semilla se sembró el 24 de febrero de 2007, día juliano $(\mathrm{dj})=55$, en charolas de poliestireno de 200 cavidades llenas con un sustrato consistente de una mezcla de "peat moss" y vermiculita. El trasplante se hizo el 30 de marzo $(\mathrm{dj})=89$, y la última cosecha se hizo el 30 de junio $(\mathrm{dj}=181)$. El arreglo de plantación fue de $1.5 \mathrm{~m}$ entre hileras y $0.45 \mathrm{~m}$ entre plantas, para una densidad de 16122 plantas ha ${ }^{-1}$. 


\section{Características físicas y químicas del suelo}

El análisis de las propiedades físicas del suelo indicó que tiene una textura franco arcillosa; densidad aparente de 1.25 y $1.36 \mathrm{~g} \mathrm{~cm}^{-3}$ a 0.1 y $0.3 \mathrm{~m}$ de profundidad respectivamente, densidad real de 2.35 a $0.1 \mathrm{~m}$ y de 2.39 $\mathrm{g} \mathrm{cm}^{-3}$ a $0.3 \mathrm{~m}$ de profundidad; porcentaje de humedad a capacidad de campo (CC) de $29.6 \%$ y a punto de marchitez permanente (PMP) de $16.5 \%$. La curva característica de humedad se determinó en el laboratorio con el método de la olla y membrana de presión, en muestras de suelo tomadas con una barrena (Uhland 1949), que luego se secaron en estufa a $105^{\circ} \mathrm{C}$ por $24 \mathrm{~h}$ con el fin de obtener el espacio poroso o humedad del suelo a saturación. Posteriormente, se determinó el contenido de humedad $(W)$ por diferencia de pesos, en función de una sucesión de cambios de succión $(\psi)$ en el proceso de secado (Figura 1). Estas características indican que es un suelo de mediana a alta capacidad de retención de humedad, con un espacio poroso o contenido volumétrico de humedad a saturación $(\theta \mathrm{s})$ que varía de 0.47 a 0.43 entre 0.1 y $0.3 \mathrm{~m}$ de profundidad.



Figura 1. Curva característica de humedad del suelo del sitio experimental.

El pH del suelo es prácticamente neutro (6.99), moderadamente pobre en materia orgánica $(1.48 \%)$, medio en nitrógeno inorgánico $\left(22.3 \mathrm{mg} \mathrm{kg}^{-1}\right)$, medio en fósforo asimilable $\left(28.79 \mathrm{mg} \mathrm{kg}^{-1}\right)$, alto en potasio aprovechable (646 $\mathrm{mg} \mathrm{kg}^{-1}$ ), moderadamente alto en calcio aprovechable $\left(2545 \mathrm{mg} \mathrm{kg}^{-1}\right)$, muy alto en magnesio aprovechable (1425 mg kg-1), medio en fierro asimilable (8.51 mg kg-1), moderadamente bajo en cobre asimilable $\left(0.79 \mathrm{mg} \mathrm{kg}^{-1}\right)$, medio en zinc asimilable $\left(1.44 \mathrm{mg} \mathrm{kg}^{-1}\right)$ moderadamente alto en manganeso asimilable $(17.58 \mathrm{mg}$ $\left.\mathrm{kg}^{-1}\right)$, y muy alto en boro asimilable $\left(2.63 \mathrm{mg} \mathrm{kg}^{-1}\right)$.

\section{Tratamientos y diseño experimental}

Se aplicaron cinco niveles de lámina de riego: 40, 60, 80,100 y $120 \%$ de la ETo, determinada con el método de
Penman-Monteith (Allen et al., 1998), y dos niveles de acolchado plástico de color plateado y negro, con y sin. Los tratamientos fueron distribuidos en un diseño completamente al azar con tres repeticiones, donde el área que riega una válvula correspondió a una lámina de riego, la cual se dividió en dos unidades experimentales, una con y otra sin cubierta plástica. La unidad experimental para cada tratamiento fue de 10 hileras de $35 \mathrm{~m}$ de longitud separadas a $1.5 \mathrm{~m}$, y con $0.45 \mathrm{~m}$ entre plantas.

El método de riego fue por goteo con cintas, cuya eficiencia de uniformidad es de $92 \%$. Las características nominales son: diámetro interno de $16 \mathrm{~mm}$, calibre 0.254 $\mathrm{mm}$, flujo de $1 \mathrm{~L} \mathrm{~h}^{-1}$, espacio entre emisores de $0.3 \mathrm{~m}, \mathrm{y}$ presión máxima de $1200 \mathrm{kPa}$.

Las características del plástico son: ancho de $1.2 \mathrm{~m}$, para una cama de $0.6 \mathrm{~m}$, calibre de $2.28 \mathrm{~mm}$, perforación parcial con diámetro de $0.063 \mathrm{~m}$ y $0.45 \mathrm{~m}$ entre espaciamiento. La instalación se hizo con una acolchadora mecánica que contiene dispositivos para construir la cama, fertilizar, y para colocar la cinta y el plástico.

Las sondas "watermark", cuyo rango de medida es de $0-200 \mathrm{kPa}$, fueron instaladas a tres profundidades $(0.1$, 0.3 y $0.4 \mathrm{~m}$ ) en cada tratamiento. Una descripción detallada sobre principios básicos de funcionamiento, construcción e instalación de las sondas, se encuentran en Thompson et al. (2006).

\section{Medición de la humedad y estimación de ETc, ETo y Kc}

Con los datos del potencial matricial medidos diariamente con las sondas 'watermark', que miden el contenido de agua en el suelo con base gravimétrica o volumétrica y cuyo valor se transforma luego a potencial mátrico con la curva característica de humedad de la Figura 1. Las sondas se instalaron a 0.1 y $0.3 \mathrm{~m}$ de profundidad en el lisímetro, y con la pérdida de humedad del suelo medida con el mismo se obtuvo una ecuación para estimar la ETc en función del potencial mátrico:

$$
\left.E T_{c}=K c K s E T_{0} \quad \text { (Ec. } 1\right)
$$

donde: $K c$ es el coeficiente de cultivo obtenido experimentalmente; $K s$ es el coeficiente de disponibilidad de agua en el suelo, que se supuso igual a uno; $E T_{0}$ es la evapotranspiración de referencia, estimada mediante la ecuación de Penman-Monteith (Allen et al., 1998) con los valores promedios de variables climáticas medidas de 6:00 a 19:00 h en una estación automática. 
La ecuación para calcular $E T_{0}$ está dada por la ecuación:

$$
E T_{0}=\frac{0.408 \Delta\left(R_{n}-G\right)+\gamma \frac{900}{T+273} u_{2}\left(e_{s}-e_{a}\right)}{\Delta+\gamma\left(1+0.34 u_{2}\right)}
$$

donde: $R_{n}$ es la radiación neta en la superficie del cultivo $\left(\mathrm{MJ} \mathrm{m}^{-2} \mathrm{~d}^{-1}\right) ; G$ es la densidad de flujo de calor del suelo $\left(\mathrm{MJ} \mathrm{m}^{-2} \mathrm{~d}^{-1}\right) ; T$ es la temperatura media diaria del aire $\left({ }^{\circ} \mathrm{C}\right) ; u_{2}$ es la velocidad del viento a $2 \mathrm{~m}$ de altura $\left(\mathrm{m} \mathrm{s}^{-1}\right)$; $e_{s}$ es la presión de vapor a saturación $(\mathrm{kPa}) ; e_{a}$ es la presión de vapor actual $(\mathrm{kPa}) ; \Delta$ es la pendiente de la curva de presión de vapor $\left(\mathrm{kPa}^{\circ} \mathrm{C}^{-1}\right)$; y $\gamma$ es la constante psicrometrica $\left(\mathrm{kPa}^{\circ} \mathrm{C}^{-1}\right)$.

Para estimar la evapotranspiración del cultivo (ETc) se utilizó un lisímetro de pesada, construido con estructura de suelo inalterada y provisto con un sistema mecánicoelectrónico. El monolito del suelo es de forma prismática, con base cuadrada de $1.8 \mathrm{~m}$ de lado y profundidad de $1.5 \mathrm{~m}$; la cara superior del monolito coincide con el ras del suelo, y la base posee un sistema de drenaje que permite la salida del agua. La precisión del sistema de pesada permite detectar cambios de peso en el lisímetro que corresponden a $0.15 \mathrm{~mm}$ de lámina de agua.

Los parámetros que pueden estimarse en los lisímetros se indican en la Ec. 3.

$$
\pm \Delta S=P+R-\left(E T_{c}+I+E\right)
$$

donde: $\Delta S$ es el cambio del contenido de humedad del suelo; $P$ es la precipitación; $R$ es el aporte del riego; ETC la evapotranspiración del cultivo; $I$ es la infiltración profunda o percolación; y $E$ es el escurrimiento superficial.

Previo al establecimiento del experimento, se calibró la pérdida de agua del suelo del lisímetro con el potencial mátrico de los primeros $0.3 \mathrm{~m}$ de profundidad, para lo cual se instalaron tensiómetros a 0.1 y $0.3 \mathrm{~m}$ de profundidad. Se evitó la incidencia de las lluvias y la calibración duró 22 d. Con base en el contenido volumétrico a saturación del suelo $\left(\theta_{s}\right)$, el cual varía de 0.43 a 0.47 , se calculó la lámina de riego a una determinada profundidad (Pr) con la Ec. 4:

$$
\left.L=\left(\theta_{s}-\theta_{0}\right) \operatorname{Pr} \quad \text { (Ec. } 4\right)
$$

donde: $\theta_{0}$ es el contenido volumétrico inicial. Con el área del lisímetro que es de $3.24 \mathrm{~m}^{2}$ se calculó el volumen de agua para saturar los primeros $0.3 \mathrm{~m}$ de profundidad, que resultó de $466 \mathrm{~L}$.
El cambio de humedad del suelo en el lisímetro se determinó mediante el registro diario del peso en el conjunto de suelo, agua y aparato. La pérdida de humedad se obtuvo por diferencia de pesos de la hora o día anterior y la hora o día actual. Las lecturas se registraron cada hora, desde las 8:00 hasta las 18:00 h.

La evapotranspiración del cultivo (ETc) se obtuvo mediante modelos generados a partir de la relación entre la pérdida de humedad en el lisímetro de pesada (Y) y el potencial mátrico (x). A la profundidad de $0.1 \mathrm{~m}: \mathrm{Y}_{1}=-$ $0.776 \mathrm{x}-1.028, \mathrm{R}^{2}=0.96$; a $0.3 \mathrm{~m}: \mathrm{Y}_{2}=-1.362 \mathrm{x}-8.89$, $R^{2}=0.92$ (Figura $2 a$ y $2 b$ ).

Puesto que cuando el contenido de humedad del suelo disminuye se requiere más energía para extraer el agua retenida, estos modelos simples estiman de manera aceptable la pérdida de humedad a partir del potencial mátrico. Tales modelos informan que por cada $\mathrm{kPa}$ de tensión, hay una pérdida promedio de humedad de $0.78 \mathrm{~mm}$ en la capa de $0.1 \mathrm{~m}$ de profundidad; y a la profundidad de 0.3 $\mathrm{m}$, la perdida de humedad es de $1.36 \mathrm{~mm}$ por cada $\mathrm{kPa}$ de tensión. Finalmente, se obtuvieron los coeficientes de cultivos por etapa fenológica, mediante la Ec. 5:

$$
K_{c}=\frac{E T_{c}}{E T_{0}} \quad(\text { Ec. 5) }
$$

Se determinó el índice de área foliar (IAF) en muestras de 3 a 10 plantas tomadas en el campo y llevadas al laboratorio, a partir de la fecha de trasplante. Los días julianos de muestreo fueron: 103, 117, 124, 138, 145, 159, 166 y 177. El área foliar se midió con un integrador de área foliar LICOR LI-3100 (LICOR, Inc. Lincoln, NE, USA).

\section{RESULTADOS Y DISCUSIÓN}

\section{Coeficientes del cultivo por etapa fenológica del cultivo}

Los coeficientes del cultivo se determinaron en el tratamiento al que se aplicaron láminas equivalentes a ETo $=100 \%$ y sin acolchado plástico. Se obtuvieron a partir de la relación entre la evapotranspiración del cultivo $(\mathrm{ETc})$ y la evapotranspiración de referencia $\left(\mathrm{ET}_{0}\right)(\mathrm{Ec} .5)$. Los valores de ETc se calcularon diariamente a partir de datos del potencial mátrico en la capa de $0.1 \mathrm{~m}$ durante la etapa vegetativa-floración y $0.3 \mathrm{~m}$ en la etapa floraciónfructificación y maduración-senescencia, profundidades donde ocurre la mayor actividad radical del cultivo en las respectivas etapas. Al sustituir estos valores en las ecuaciones lineales generadas en el lisímetro de pesada para estimar ETc, se obtuvieron los coeficientes de cultivo por etapa fenológica (Cuadro 1). 

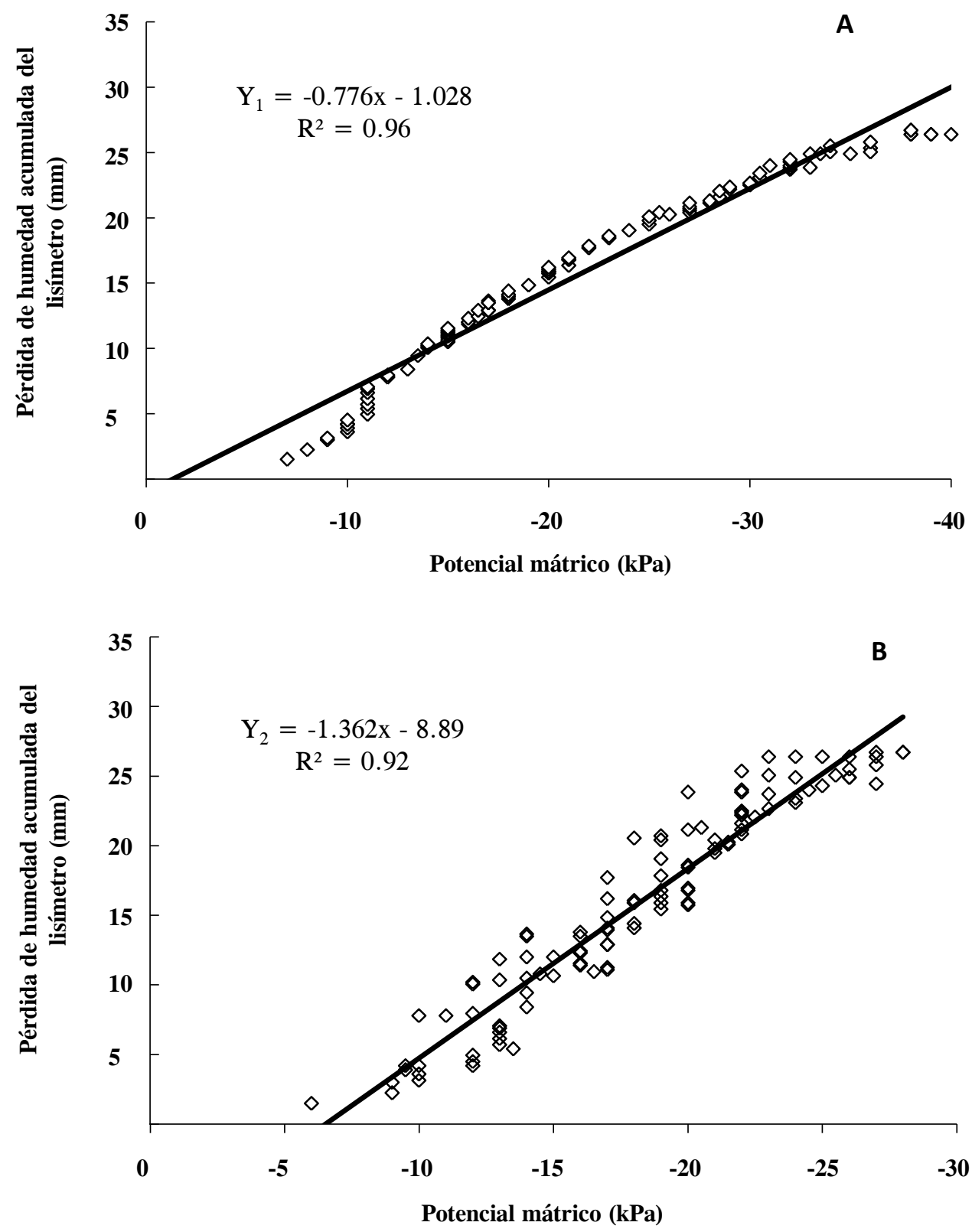

Figura 2. Relación entre el potencial mátrico a $0.1 \mathrm{~m}$ (A) y $0.3 \mathrm{~m}$ de profundidad (B) y la pérdida de humedad del lisímetro en $\mathrm{mm}$, durante 22 días. Cada punto representa la pérdida de agua medida cada $2 \mathrm{~h}$ entre las 8:00 y las 18:00 h.

Cuadro 1. Coeficientes de cultivo de tomate de cáscara en diferentes etapas fenológicas clasificadas en días después del trasplante, sin acolchado plástico y con riego por goteo.

\begin{tabular}{lcrrr}
\hline Etapa & Días & \multicolumn{1}{c}{$\begin{array}{c}\text { ETc } \\
(\mathrm{mm})\end{array}$} & \multicolumn{1}{c}{$\begin{array}{c}\text { ET0 } \\
(\mathrm{mm})\end{array}$} & $\mathrm{Kc}$ \\
\hline Vegetativa-Floración & 45 & 50.20 & 165.60 & 0.30 \\
Floración-Fructificación & 35 & 121.50 & 111.96 & 1.10 \\
Maduración-Senescencia & 20 & 71.36 & 82.92 & 0.86 \\
\hline
\end{tabular}

Los valores de Kc aquí estimados son similares a los propuestos por Allen et al. (1998) para el cultivo de tomate (Lycopersicon esculentum L.) sin cubierta plástica, excepto en la etapa inicial donde se encontró un valor más alto de 0.6. En las curvas acumuladas de ETc y ETo (Figura 3) se nota que la $\mathrm{ET}_{0}$ tiene un comportamiento lineal durante el crecimiento del cultivo, mientras que la ETc no da una curva lineal y generalmente presenta valores menores que la $\mathrm{ET}_{0}$, y que estas tasas de evapotranspiración dependen de las condiciones de manejo del cultivo y de la etapa fenológica. La evapotranspiración del cultivo es siempre diferente a la del cultivo de referencia $\left(\mathrm{ET}_{0}\right)$, debido a las diferencias en cobertura del suelo, 
propiedades de la vegetación y resistencia aerodinámica, con respecto a la del pasto (Allen et al., 1998). Los efectos de las características del cultivo en estudio están incorporados en el coeficiente de cultivo. En la etapa inicial del crecimiento, el efecto principal es la evaporación del suelo, especialmente en la parte expuesta del mismo, y la humedad de la superficie del suelo que tiene influencia sobre el valor de la resistencia superficial, que es la suma de la resistencia del suelo y la resistencia de las raíces. Inmediatamente después de humedecer el suelo a través del riego o lluvia, la tasa de transferencia de vapor de agua desde el suelo es alta.

El coeficiente del cultivo $(\mathrm{Kc})$ incluye los efectos de la evaporación de la superficie del suelo y del cultivo y depende de la disponibilidad de agua en el suelo en la zona radical y de la menos húmeda de la superficie expuesta del suelo. Estos valores se obtienen de cultivos regados en forma adecuada, sin estrés hídrico. También se pueden usar otras variables de tiempo, como los grados días de desarrollo, unidades térmicas-solares, o $\mathrm{ET}_{0}$ acumulativa (DeTar, 2004; Bandyopadhyay et al., 2005; DeTar, 2009).

Los valores de coeficientes único (promedio temporal) del cultivo $(\mathrm{Kc})$ de tomate no estresado con manejo adecuado y altura de $0.6 \mathrm{~m}$, en clima subhúmedo con humedad relativa mínima $\left(\mathrm{HR}_{\min }=45 \%\right)$, y velocidad del viento $\mathrm{u}=2 \mathrm{~m} \mathrm{~s}^{-1}$, se usaron en la ecuación de Penman-Monteith para estimar la ETo del tomate de cascara. Allen et al. (1998) proponen valores de Kc para las etapas inicial, intermedia y final de $0.6,1.15$ y de 0.7 a 0.90 , respectivamente; aclaran que cuando el cultivo crece de 1.5 a $2 \mathrm{~m}$ de altura, puede utilizarse un $\mathrm{Kc}$ de 1.2 durante la etapa intermedia.



Figura 3. Evapotranspiración acumulada de ETc y ETo en el cultivo de tomate de cáscara sin acolchado plástico y con riego por goteo. 


\section{Determinación de Kc para el cultivo con cubierta de plástico}

La cobertura plástica con riego por goteo reduce significativamente la evaporación del agua en la superficie del suelo. Asociada con la reducción de la evaporación, hay un incremento general de la transpiración del cultivo debido a la transferencia de calor sensible y radiactivo desde la superficie de la cobertura plástica hacia el dosel del cultivo. Aunque la tasa de transpiración con plástico puede incrementarse en un promedio de 10-30 \% durante el ciclo, comparado con la ausencia de la cobertura plástica, el valor de Kc en promedio disminuye de 10-35 \% debido a la reducción de la evaporación en el suelo (Allen et al., 1998).

Para obtener los coeficientes de cultivo con riego por goteo y con acolchado plástico, si se considera la reducción aproximada de $35 \%$ de los valores de Kc para el cultivo de tomate con cobertura plástica en comparación con la condición sin cobertura (Allen et al., 1998), los Kc para tomate de cáscara pueden ser de 0.2, 0.71 y 0.56 para las etapas vegetativa-floración, floración-maduración y maduración-senescencia, respectivamente.

Los coeficientes específicos y el índice de área foliar son valores importantes para lograr un mejor aprovechamiento de los recursos hídricos. En las Figuras 4 y 5 se presenta la relación del $\mathrm{Kc}$ con el índice de área foliar $(I A F)$ evaluado después del trasplante del cultivo sin acolchado (Ec. 6) y con acolchado plástico (Ec. 7), en condiciones de máxima disponibilidad de agua:

$$
\begin{array}{r}
K c=0.6569(I A F)+0.2404, \\
\mathrm{R}^{2}=0.96, \quad \mathrm{n}=8, \quad \mathrm{P}<0.01 \\
\\
K c=0.4144(I A F)+0.1691, \\
\mathrm{R}^{2}=0.93, \quad \mathrm{n}=8, \quad \mathrm{P}<0.01
\end{array}
$$

Estas relaciones indican que el Kc puede estimarse a partir del IAF del tomate de cáscara. Según estos resultados, cuando el IAF es igual a uno el coeficiente del cultivo tiende a aproximarse a la unidad en ausencia de acolchado plástico (Figura 4), e incluso tiende a ser mayor que uno, lo cual indica que la ETc supera a la ETo. Sin embargo, para el cultivo de cacahuate (Arachis hypogaea L.) Bandyopadhyay et al. (2005) determinaron que cuando el IAF es mayor que tres, el valor del $\mathrm{Kc}$ es mayor que uno.

Los resultados aquí obtenidos también demostraron que el acolchado plástico reduce los coeficientes de cultivo y por ende, la evapotranspiración del cultivo, sobre todo en las etapas de floración y de maduración, lo que se traduce en un ahorro de agua en la programación del riego del cultivo.



Figura 4. Coeficientes de cultivo en tomate de cáscara en función del índice de área foliar sin acolchado plástico y riego por goteo. 




Figura 5. Coeficientes de cultivo en tomate de cáscara en función del índice de área foliar, con acolchado plástico y riego por goteo.

El método del cálculo del IAF y el modelo de PenmanMonteith para evaluar la resistencia del cultivo simplifica los complejos procesos de la evapotranspiración; además, resulta preciso y confiable para evaluar la productividad del agua y de los cultivos cubiertos con acolchado plástico (Lovelli et al., 2008; Li et al., 2008).

\section{Efecto de la lámina de riego y acolchado plástico en el potencial mátrico del suelo}

En las Figuras 6, 7 y 8 se muestran los resultados del efecto de las láminas de riego, con reposición de 40, 60 y $100 \%$ de la ETo respectivamente, así como del acolchado plástico, sobre el potencial mátrico a diferentes profundidades del suelo. Las cantidades totales del agua de riego aplicadas fueron: 154, 208, 263, 317 y $371 \mathrm{~mm}$, correspondientes a 40, 60, 80, 100 y $120 \%$ de la ETo. En los tratamientos de 40 y $60 \%$ no se presentan las gráficas de $0.4 \mathrm{~m}$ de profundidad debido a que durante la mayor parte del ciclo del cultivo, las sensores 'watermark' registraron potenciales mátricos menores que $-80 \mathrm{kPa}$.

Se detectaron diferencias en valores del potencial mátrico del suelo entre profundidades, debido al efecto de la lámina de riego y de la lluvia. Los valores del potencial mátrico en los sistemas con acolchado plástico son mayores que en los tratamientos sin acolchado, aunque durante el ciclo del cultivo se presentaron lluvias que influyeron en la humedad del suelo, sobre todo en los tratamientos sin cubierta plástica. Debido a los eventos de lluvia ocurridos durante el ciclo del cultivo, los valores del potencial son mayores en los tratamientos sin acolchado durante estos días, como los ocurridos en los días julianos (dj): 119, 139, 146 y 160, correspondientes a las fechas de 29 de abril, 19 y 26 de mayo y 9 de junio, respectivamente. Dichos potenciales aumentaron en la capa de $0.1 \mathrm{~m}$ de profundidad, principalmente en los tratamientos de $40 \%$ de la ETo (Figura 6).

A 0.1 y $0.3 \mathrm{~m}$ de profundidad, cuando se aplicó $40 \%$ de la $\mathrm{ET}_{0}$ el potencial mátrico durante la mayor parte del ciclo del cultivo fue menor que $-80 \mathrm{kPa}$, tanto en sistemas con y sin acolchado plástico; este valor es considerado deficitario y con efectos negativos en el rendimiento de frutos (López et al., 2009), especialmente si estas condiciones de alta tensión se mantienen por periodos prolongados (Gratacos y Gurovich, 2003).

Los valores del potencial mátrico del suelo están directamente relacionados con el régimen de riego establecido. En los tratamientos donde se repuso $40 \%$ de la $\mathrm{ET}_{0}$, tanto en los tratamientos con y sin acolchado plástico, los potenciales alcanzaron valores menores que -80 $\mathrm{kPa}$ principalmente en la etapa de floración y de maduración del cultivo (Figura 6), lo que indica que esa lámina de riego es insuficiente para mantener niveles de humedad convenientes para el crecimiento del cultivo y el rendimiento de frutos. Sin embargo, al considerar la 
productividad o uso eficiente del agua, el acolchado plástico representa mejor opción que la ausencia de acolchado (López et al., 2009).

Durante la etapa inicial o vegetativa, con su régimen de riego equivalente a $60 \%$ de la $\mathrm{ET}_{0}$ es suficiente para mantener niveles de humedad cercanos a capacidad de campo $(-30 \mathrm{kPa})$ en los primeros $0.3 \mathrm{~m}$ de profundidad, tanto en los tratamientos de acolchado como sin acolchado. A medida que el cultivo alcanza la etapa de floración, el potencial mátrico del suelo disminuye aun más donde no existe acolchado plástico, en las profundidades de 0.1 y $0.3 \mathrm{~m}$ (Figura 7). Esto se debe a que el plástico reduce o elimina la evaporación del suelo, aunque en la etapa de floración, sin eventos de lluvias, los niveles de tensión fueron menores que $-80 \mathrm{kPa}$. En la etapa final del cultivo las lluvias contribuyeron a que aumentaran los niveles de humedad en el suelo y que el potencial mátrico se aproximara a capacidad de campo ($30 \mathrm{kPa}$ ), principalmente el día 160 (9 de junio) cuando ocurrió una precipitación de $40 \mathrm{~mm}$.

Los tratamientos de $100 \%$ de la $\mathrm{ET}_{0}$, tanto sin acolchado como con acolchado plástico, mantienen niveles de potencial mátrico cerca de capacidad de campo $(<30 \mathrm{kPa})$ en la etapa vegetativa, hasta $0.3 \mathrm{~m}$ de profundidad del suelo (Figura 8). Pero a finales de la floración, la tensión de humedad alcanzó valores que llegan hasta $-80 \mathrm{kPa}$, lo que originó que el Kc resultara mayor que uno.

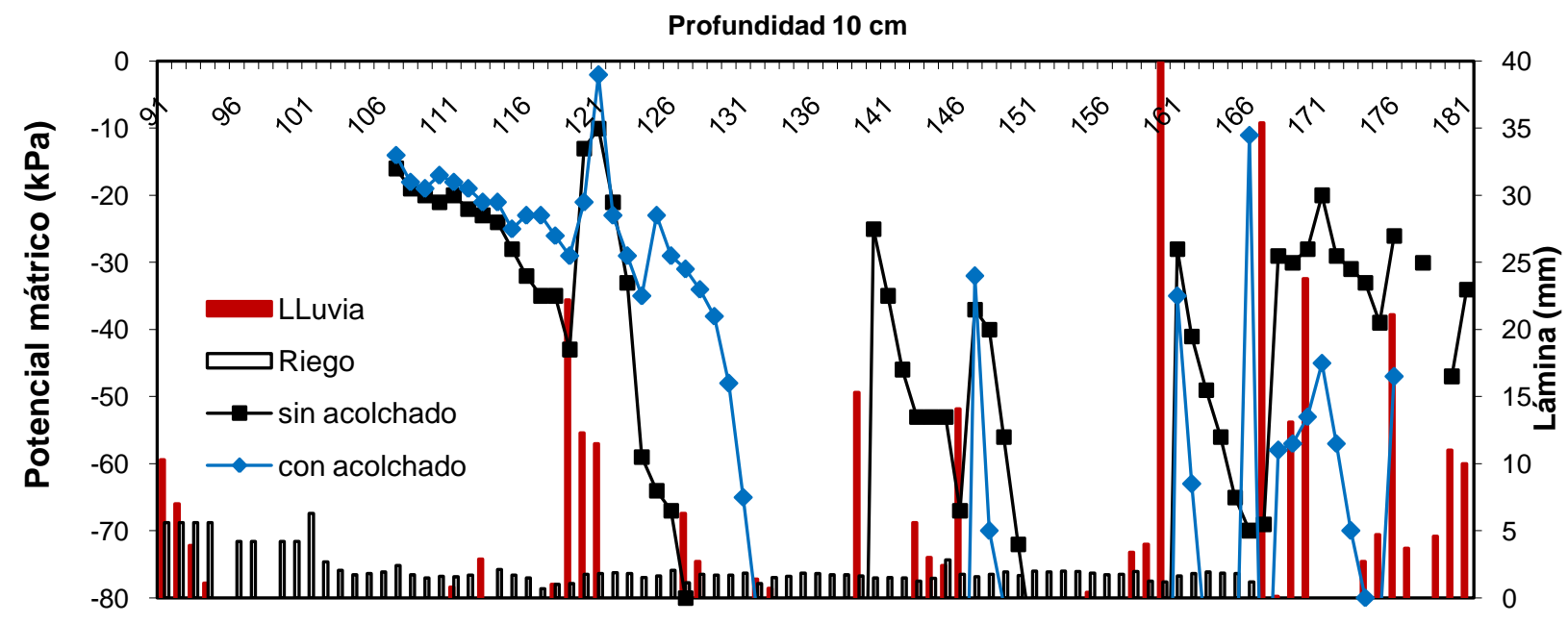

Profundidad $30 \mathrm{~cm}$

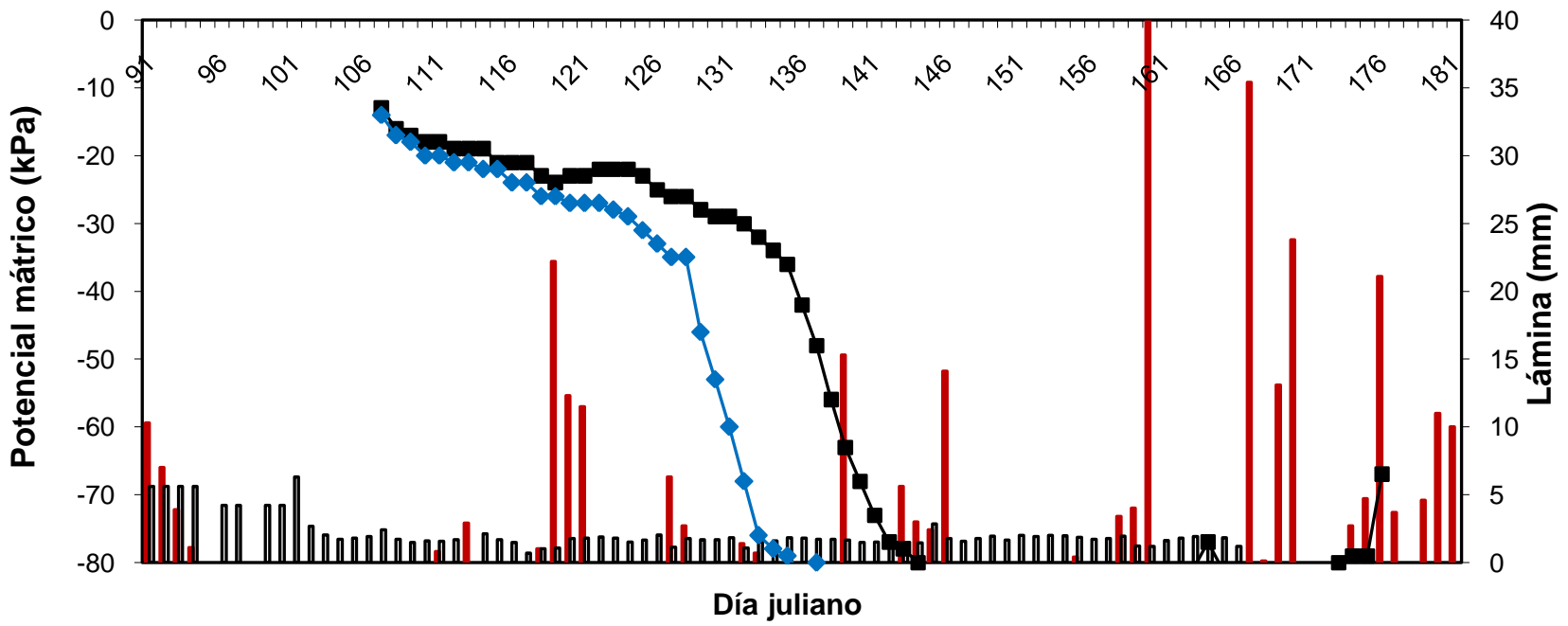

Figura 6. Efecto de la lámina de riego y lluvia en los tratamientos de $40 \%$ de la evapotranspiración de referencia (ET0), con y sin acolchado, en el potencial mátrico a dos profundidades del suelo $(10 \mathrm{y} 30 \mathrm{~cm})$. 
Para que los sensores de humedad del suelo sean adecuados en la programación del riego en cultivos hortícolas con riego por goteo, éstos deben de ser capaces de proporcionar datos precisos en suelos relativamente húmedos $(-10$ a $-30 \mathrm{kPa})$ en condiciones dinámicas de frecuentes ciclos de humedecimiento y secado y en condiciones de suelos secos $(<-30 \mathrm{kPa})$. Las medidas del potencial mátrico en los primeros $0.3 \mathrm{~m}$ de profundidad constituyen un buen indicador de la disponibilidad de agua en el suelo, y una herramienta útil para el monitoreo del riego en cultivo. La diferencia encontrada en las medidas de tensión de humedad en las profundidades de 0.1, $0.3 \mathrm{y}$ $0.4 \mathrm{~m}$, indica la importancia de elegir correctamente la profundidad donde se instalen las sondas "watermark" que deben estar en la zona radical más activa, en cada etapa fenológica del cultivo. Asenjo y Yuste (2003) afirman que el conocimiento amplio del suelo y de la distribución del sistema radical del cultivo, son elementos básicos para el correcto manejo del riego.
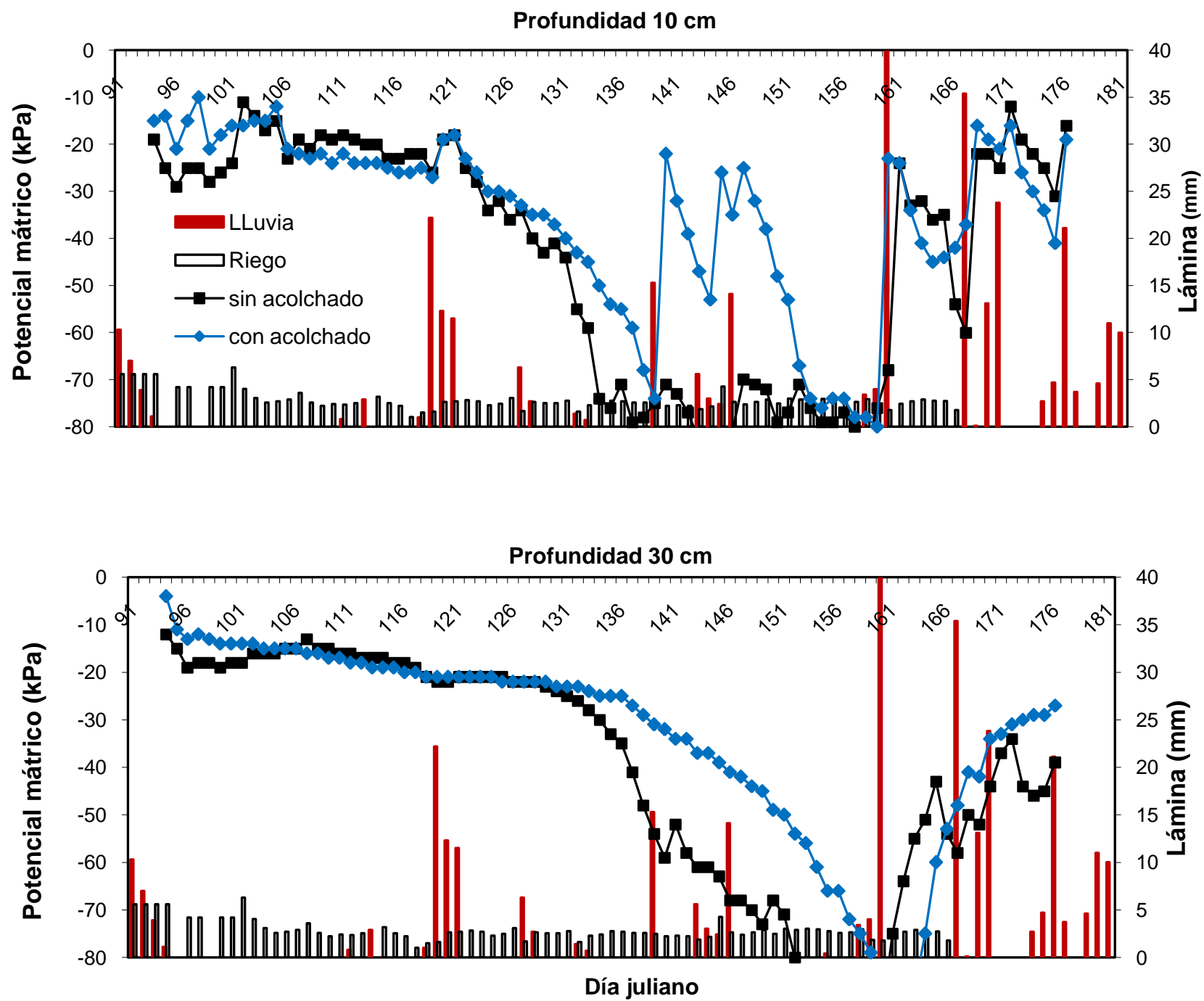

Figura 7. Efecto de la lámina de riego y lluvia en los tratamientos de $60 \%$ de la evapotranspiración de referencia (ET), con y sin acolchado, en el potencial mátrico a dos profundidades del suelo $(10 \mathrm{y} 30 \mathrm{~cm})$. 

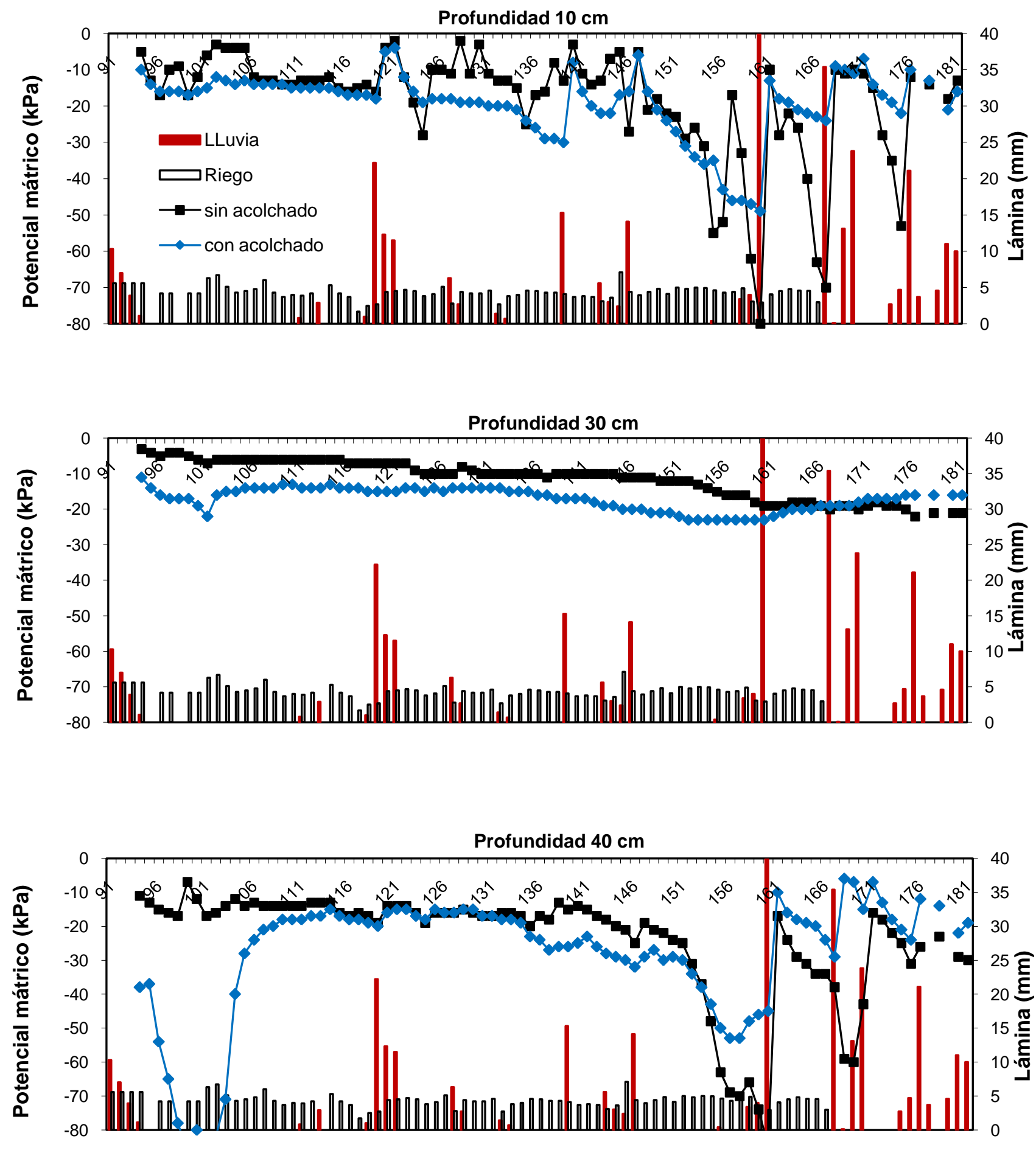

Día juliano

Figura 8. Efecto de la lámina de riego y lluvia en los tratamientos de $100 \%$ de la evapotranspiración de referencia (ET), con y sin acolchado, en el potencial mátrico a tres profundidades del suelo $(10,30$ y $40 \mathrm{~cm})$. 


\section{CONCLUSIONES}

Las mediciones diarias del potencial mátrico permiten estimar de manera confiable las pérdidas de humedad en el suelo, así como calcular la evapotranspiración del cultivo. Los coeficientes de cultivo (Kc) del tomate de cáscara crecido sin cobertura plástica fueron: en etapa vegetativa de 0.3 , de 1.1 en floración y de 0.86 en maduración. Cuando el cultivo se cubre con acolchado plástico, los coeficientes de cultivo se disminuyen a 0.2 , 0.71 y 0.56 para las etapas vegetativa, floración y maduración, respectivamente. Los $K c$ para el tomate de cáscara sin acolchado pueden estimarse a partir del índice de área foliar $(I A F)$ mediante la ecuación $K c=$ $0.6569(I A F)+0.2404$, y con acolchado plástico mediante la ecuación $K c=0.4144(I A F)+0.1691$, y en condiciones de máxima disponibilidad de agua. El potencial mátrico del suelo varía entre profundidades debido al efecto de la lámina de riego o lluvia y el acolchado plástico, y son mayores cuando el cultivo se cubre con acolchado plástico que sin acolchado.

\section{BIBLIOGRAFÍA}

Allen G R, S L Pereira, D Raes, M Smith (1998) Crop Evapotranspiration. FAO Irrigation an Drainage Paper No. 56. FAO, Rome, Italy. $300 \mathrm{p}$.

Arteaga R R, M A Vázquez, P Coras, V Ángeles (2006) Componentes de la estación de crecimiento, variación temporal y espacial en Chapingo, México. Ingeniería Hidráulica en México. 21:57-68.

Asenjo L J, J Yuste (2003) Estimación del estado hídrico del suelo por tensiometría y volumetría y su relación con el estado hídrico del viñedo. In: Estudios de la Zona No Saturada del Suelo Vol. VI. J Álvarez-Benedí, P Marinero (eds). Disponible en: www.zonanosaturada.com/publics/V6/p177-180.pdf (Febrero 2009).

Bandyopadhyay P K, S Mallick, S K Rana (2005) Water balance and crop coefficients of summer-grown peanut (Arachis hypogaea L.) in a humid tropical region of India. Irrig. Sci. 23:161169.

Burman R, L O Pochov (1994) Evaporation, Evapotranspiration and Climatic Data. Developments in Atmospheric Science, 22. Elsevier Science B.V. U.S.A. 278 p.
Catalán V E A, M M Villa (2009) Sistema experto para el manejo eficiente del riego parcelario. Red Tematica de Agua del Consejo Nacional de Ciencia y Tecnología. Disponible en: http://red-tematica-conacyt.blogspot.com/2009/05/sistemaexperto-para-el-manejo.html (Mayo 2009).

DeTar W R (2004) Using a subsurface drip irrigation system to measure crop water use. Irrig. Sci. 23:111-122.

DeTar W R (2009) Crop coefficients and water use for cowpea in the San Joaquin Valley of California. Agric. Water Manage. 96:53-66

Dodds E P, S M Wayne, A Barton (2005) A Review of Methods to Estimate Irrigated Reference Crop Evapotranspiration across Australia. CRC for Irrigation Futures Technical Report No. 04/05. CSIRO Land and Water. Disponible en www.clw.csiro.au/publications/consultancy/2005 (Abril 2005).

Doorenbos J, W O Pruitt (1977) Guidelines for Predicting Crop Water Requirements. FAO Irrigation and Drainage Paper No. 24. F.A.O., Rome. 144 p.

Gratacos E, L Gurovich (2003) Uso de la técnica del fitomonitor como indicador del estado hídrico del kiwui y su uso en riego programado. Cien. Inv. Agr. Chile. 30:113-137.

Hillel D (1980) Fundamentals of Soils Physics. Academic Press, Inc. New York. 413 p.

Jensen M E, J L Wright (1978) The role of evapotranspiration models in irrigation scheduling. Trans. ASAE. 21:82-87.

Jensen M E (1981) Sumary and challenges. In: Irrigation Scheduling for Water and Energy Conservation in the80's. Proc. ASAE, Irrigation Scheduling Conference. ASAE. P.O. Box 410. St. Joseph, Michigan. pp: 225-231.

Li S, S Kang, F Li, L Zhang (2008) Evapotranspiration and crop coefficient of spring maize with plastic mulch using eddy covariance in northwest China. Agric. Water Manage. 95:1214-1222.

López L R, R Arteaga, M A Vázquez, I L López, I Sánchez (2009) Producción de tomate de cáscara (Physalis ixocarpa Brot) basado en láminas de riego y acolchado plástico. Rev. Chapingo S. Hort. 15:83-89.

Lovelli S, M Perniola, M Arcieri, A R Rivelli, T. Di Tommaso (2008) Water use assessment in muskmelon by the PenmanMonteith "one-step" approach. Agric. Water Manage. 95:1153-1160

Lubana PP S, NK Narda, S Thaman (2001) Performance of summer planted bunch groundnut under different levels of irrigation. Indian J. Agric. Sci. 71:783

Thompson R B, M Gallardo, T Agëera, L C Valdez, M D Fernández (2006) Evaluation of the Watermark sensor for use with drip irrigated vegetable crops. Irrig. Sci. 24:185-202.

Uhland R E (1949) Physical properties of soils as modiefied by crops and management. Soil Science. Society of America Proceedings, 14: 361-366. 